\title{
Toll-Like Receptor 4 on Nonhematopoietic Cells Sustains CNS Inflammation during Endotoxemia, Independent of Systemic Cytokines
}

\author{
Sumana Chakravarty and Miles Herkenham \\ Section on Functional Neuroanatomy, National Institute of Mental Health, National Institutes of Health, Bethesda, Maryland 20892
}

\begin{abstract}
Inflammatory agonists such as lipopolysaccharide (LPS) induce robust systemic as well as CNS responses after peripheral administration. Responses in the innate immune system require triggering of toll-like receptor 4 (TLR4), but the origin of CNS sequelas has been controversial. We demonstrate expression of TLR4 transcripts in mouse brain in the meninges, ventricular ependyma, circumventricular organs, along the vasculature, and in parenchymal microglia. The contribution of TLR4 expressed in CNS resident versus hematopoietic cells to the development of CNS inflammation was examined using chimeric mice. Reciprocal bone marrow chimeras between wild-type and TLR4 mutant mice show that TLR4 on CNS resident cells is critically required for sustained inflammation in the brain after systemic LPS administration. Hematopoietic TLR4 alone supported the systemic release of acute phase cytokines, but transcription of proinflammatory genes in the CNS was reduced in duration. In contrast, TLR4 function in radiation-resistant cells was sufficient for inflammatory progression in the brains of chimeric mice, despite the striking absence of cytokine elevations in serum. Surprisingly, a temporal rise in serum corticosterone was also dependent on TLR4 signaling in nonhematopoietic cells. Our findings demonstrate a requirement for TLR4 function in CNS-resident cells, independent of systemic cytokine effects, for sustained CNS-specific inflammation and corticosterone rise during endotoxemia.
\end{abstract}

Key words: inflammation; neuroimmunology; lipopolysaccharide; rodent; blood-brain; corticosterone

\section{Introduction}

Systemic endotoxin is associated with the development of septicemia in clinical settings. In experimental models, bacterial lipopolysaccharide (LPS) closely mimics the consequences of sepsis: offsetting thermoregulatory balance to cause fever, activating the hypothalamic-pituitary-adrenal (HPA) axis to release glucocorticoids, and modulating CNS centers controlling behaviors associated with locomotor activity, thirst, appetite, social interactions, and motivational status. Interestingly, these physiological changes also represent the CNS-mediated components of an acute-phase response to systemic infection and reflect the similarity in central neural circuits that are affected in diverse clinical and experimental scenarios.

A large part of the response of the brain to LPS has been attributed to the action of cytokines and other acute phase reactants, but the origin of inflammatory mediators has remained controversial. Because receptors for LPS are highly expressed on innate immune cells, it is conceivable that endotoxin recognition

\footnotetext{
Received 0ct. 13, 2004; revised Dec. 20, 2004; accepted Dec. 25, 2004.

This work was supported by the Intramural Research Program of the National Institute of Mental Health. We thank Eileen Briley for expert technical assistance.

Correspondence should be addressed to Dr. Miles Herkenham, Section on Functional Neuroanatomy, National Institute of Mental Health, Building 35, Room 1C913, 35 Convent Drive, Bethesda, MD 20892-3724. E-mail: herkenh@mail.nih.gov.

S. Chakravarty's present address: Department of Molecular Microbiology and Immunology, Bloomberg School of Public Health, Johns Hopkins University, 615 North Wolfe Street, Baltimore, MD 21205.

DOI:10.1523/JNEUROSCI.4268-04.2005

Copyright $\odot 2005$ Society for Neuroscience $\quad 0270-6474 / 05 / 251788-09 \$ 15.00 / 0$
}

by hematopoietic cells triggers the systemic release of secondary mediators that in turn impinge on the brain. Indeed, peripheral administration of cytokines can elicit CNS-mediated acute-phase response components that are the same as those elicited by LPS (Duff and Durum, 1983; Berkenbosch et al., 1987; Laflamme and Rivest, 1999). Alternatively, the CNS response during endotoxemia can be initiated locally as a result of inflammatory activation within the brain (Breder et al., 1994; Breder and Saper, 1996; Elmquist et al., 1996; Quan et al., 1997, 1999; Ivanov et al., 2002; Reyes et al., 2003; Rivest, 2003). With regard to generation of the innate immune response of the brain, therefore, it is unclear whether LPS directly activates signal transduction in CNS cells. Expression of toll-like receptor 4 (TLR4), the signaling receptor for LPS (Poltorak et al., 1998), in meninges, choroid plexus, and circumventricular organs (CVOs) of the rat brain (Laflamme and Rivest, 2001) and induction of proinflammatory transcripts like interleukin-1 $\beta$ (IL-1 $\beta$ ), tumor necrosis factor $\alpha(\mathrm{TNF} \alpha)$, inhibitory factor $\kappa \mathrm{B} \alpha(\mathrm{I} \kappa \mathrm{B} \alpha)$, and $\mathrm{CD} 14$ at similar sites provide for the possibility of direct TLR4-mediated LPS action in the CNS.

Our study reports a high-resolution in situ analysis of TLR4 mRNA distribution in the mouse brain. Although TLR4 expression has been reported in cultured primary microglia (Bsibsi et al., 2002; Lehnardt et al., 2002), our analysis allowed us to examine the anatomical specificities of TLR4-bearing CNS cells in situ. We show that the transcript is more widely expressed in bloodbrain barrier-associated and parenchymal brain cells than previously recognized (Laflamme and Rivest, 2001). To further ad- 
dress the functional consequence of TLR4 activation in resident cells during endotoxemia, we generated bone marrow (BM) chimeras using wild-type (WT) and TLR4 mutant HeJ mice that are hyporesponsive to LPS. The strategy allowed us to restrict LPS responsiveness to either the hematopoietic or nonhematopoietically derived tissue and demonstrate that LPS-TLR4 interaction in nonhematopoietic-derived cells is needed to perpetuate an intra-CNS inflammatory response and one physiological hallmark of the acute phase response: increased plasma corticosterone levels. Our findings highlight the presence of the LPS signaling receptor in resident cells of the mouse brain and suggest its critical participation in shaping the CNS response to circulating endotoxin.

\section{Materials and Methods}

Animals. Male C57BL/6, C3H/HeJ, C3H/HeOuJ, and $\mathrm{C} 57 \mathrm{BL} / 10 \mathrm{ScCr}$ mice were purchased from The Jackson Laboratory (Bar Harbor, ME) at 4-6 weeks of age. They were housed at the National Institute of Mental Health animal facility. All procedures conformed to National Institutes of Health guidelines regarding the care and use of animals and were conducted under an Animal Care and Use Committee-approved protocol.

LPS repurification. LPS serotype 055:B5 was purchased from Sigma (St. Louis, MO). Commercial LPS was repurified exactly as described previously (Manthey et al., 1994). A $5 \mathrm{mg} / \mathrm{ml}$ solution of LPS in endotoxin-free water containing $0.2 \%$ triethylamine (TEA) was treated with deoxycholate (DOC) to a final concentration of $0.5 \%$, followed by phenol extraction with an equal volume of water-saturated phenol. The mixture was re-extracted twice with $0.2 \%$ TEA and $0.5 \%$ DOC, the aqueous phases were pooled, adjusted to $75 \%$ ethanol and $30 \mathrm{~mm}$ sodium acetate, and they were allowed to precipitate at $-20^{\circ} \mathrm{C}$ for $1 \mathrm{~h}$. The precipitates were washed in cold $100 \%$ ethanol, air-dried, weighed, and dissolved in endotoxin-free saline at a concentration of $5 \mathrm{mg} / \mathrm{ml}$.

LPS injections. Mice were injected intraperitoneally with $1 \mathrm{mg} / \mathrm{kg}$ LPS (commercial or repurified) between 7:00 and 8:00 A.M. and killed 2 or $6 \mathrm{~h}$ later. Brain and spleen were dissected out and rapidly frozen. Serum was extracted and stored along with the frozen tissue at $-70^{\circ} \mathrm{C}$.

Constructing chimeric mice. Recipient mice were exposed to a single dose of 9 grays. Bone marrow was flushed from the femurs and tibias of donor mice, and 1-4 $\times 10^{6}$ cells were injected intravenously into irradiated recipients. After 12 weeks of reconstitution, chimeric mice were either killed to assess reconstitution efficiency or injected with LPS as described.

Fluorescence-activated cell sorter analysis. Single-cell suspensions from spleens of chimeric mice were labeled with antibodies to CD45 as well as antibodies specific to the major histocompatibility complex (MHC) class I molecules $(\mathrm{H}-2 \mathrm{~K})$ of the $\mathrm{k}\left(\mathrm{H}-2 \mathrm{~K}^{\mathrm{k}}\right)$ or b $\left(\mathrm{H}-2 \mathrm{~K}^{\mathrm{b}}\right)$ haplotype according to standard protocols. Staining was analyzed on a BD FACScalibur cell sorter. Antibodies were purchased from BD PharMingen (San Diego, CA).

ELISA on serum samples. Cytokines were detected in mice sera using quantikine ELISA kits (R \& D Systems, Minneapolis, MN) for mouse interferon $\gamma$ (IFN $\gamma$ ) (catalog \#MIF00), IL-1 $\beta$ (catalog \#MLB00), TNF $\alpha$ (catalog \#MTA00), and IL-12p70 (catalog \#M1270) according to manufacturer's instructions.

RIA on serum samples. Corticosterone levels were assayed in serum samples using a radioimmunoassay kit from MP Biomedicals (Irvine, CA) according to manufacturer's instructions.

In situ hybridization and immunohistochemistry. Full-length mTLR4 cDNA was cloned from total RNA isolated from mouse spleen. Reversetranscribed cDNA was amplified by PCR with primers against the $5^{\prime}$ (bases 1-15) and 3' (bases 2491-2505) ends of the complete open reading frame of TLR4 (GenBank accession number AF185285). EcoRI and SalI sites (highlighted in bold) were engineered into the primer sequences (TLR4F, 5'-GGATCCATGATGCCTCCCTGG-3'; TLR4R, $3^{\prime}$ TGCCGTTGAACCTGGCAGCTG-5' ${ }^{\prime}$ ). The $2.5 \mathrm{~kb}$ PCR product was cloned into pGEM-Teasy (Promega, Madison, WI). Full-length CD14 and a fragment of TLR2 were similarly cloned using the following prim- ers (CD14F, GGATCCATGGAGCGTGTGCTT; CD14R, CTCTAGCGGAGAAACAACAGCTG; TLR2F， AGACACTGGGGGTAACATCG; TLR2R, TCAGGAAGCGCATCTTTTCT). Full-length $1.05 \mathrm{~kb} \mathrm{I \kappa B} \alpha$ cDNA was a gift from Rebecca Taub (University of Pennsylvania, Philadelphia, PA), and mouse IL- $1 \beta$ cDNA was generously provided by Serge Rivest (Laval University, Quebec, Canada). A full-length TNF $\alpha$ containing clone (clone number 63169) was purchased from American Type Culture Collection (Manassas, VA). Radioactive antisense probes were transcribed using the Riboprobe System (Promega) with the appropriate RNA polymerase and $\left[{ }^{35} \mathrm{~S}\right] \mathrm{UTP}$ (specific activity $>1000 \mu \mathrm{Ci} /$ mmol; NEN, Boston, MA). Nonradioactive probes were labeled using a digoxigenin (DIG) labeling mix (Roche Diagnostics, Indianapolis, IN).

In situ hybridization was performed as described previously (Whitfield et al., 1990). After standard prehybridization procedures, sections were hybridized with ribonucleotide probes radiolabeled with ${ }^{35} \mathrm{~S}$ at a concentration of $5 \times 10^{6} \mathrm{cpm} /$ section overnight at $55^{\circ} \mathrm{C}$, given a final $1 \mathrm{~h}$ wash of $0.2 \times$ SSC at $60^{\circ} \mathrm{C}$, dehydrated with ethanol, and air-dried for autoradiography.

Some sections were processed for double-label in situ hybridization and immunohistochemistry, using ${ }^{35}$ S-labeled TLR4 mRNA and an antibody against the neuronal marker neuronal-specific nuclear protein (NeuN; Chemicon, Temecula, CA), microglial marker Ibal (a gift from Dr. Y. Imai, National Institute of Neuroscience, Tokyo, Japan), astrocyte marker glial fibrillary acidic protein (GFAP; Dako, Carpinteria, CA), or biotin-conjugated isolectin B4 (Molecular Probes, Eugene, OR). The hybridization procedure was applied first as described above, with the following modification. For Ibal and GFAP double-labeling, slides were initially fixed at $\mathrm{pH} 9.5$ for $1 \mathrm{~h}$. Hybridization was followed by standard immunohistochemistry procedures. Before air-drying slides for autoradiography, sections were incubated in a $1.5 \%$ blocking solution (DIG labeling kit; Roche Diagnostics), followed by primary and secondary antibodies. The signal was amplified using Vector $\mathrm{ABC}$ reagents and visualized with DAB (Vector Laboratories, Burlingame, CA).

Dual-label in situ hybridization procedures combined digoxigeninlabeled CX3CR1 cRNA with ${ }^{35} \mathrm{~S}$-labeled TLR4 cRNA. The two probes were hybridized simultaneously as described previously (Hohmann and Herkenham, 1999) with omission of dehydration and delipidation steps before hybridization. Double-label slides were emulsion-coated in LM-1 emulsion (Amersham Biosciences, Piscataway, NJ). Single-label slides were dipped in NTB-2 emulsion (Eastman Kodak, Rochester, NY). Both were exposed, developed (D-19; Eastman Kodak), and counterstained with cresyl violet or methyl green. Slides and ${ }^{14} \mathrm{C}$ plastic standards (American Radiochemicals, St. Louis, MO) were placed in x-ray cassettes, apposed to film (BioMax MR; Eastman Kodak), and developed (X-OMAT; Eastman Kodak). Autoradiographic film images of brain sections and standards were digitized with NIH Image software (http:// rsb.info.nih.gov/nih-image/index.html). Quantification was performed by a previously described method (Wang and McGinty, 1995; Adams et al., 2000) using the density slice feature. The upper limit of the density slice was set at the maximal absorbance value (255) for all sections, and the lower limit of the density slice was adjusted to eliminate background. The hybridization signal for a particular riboprobe was measured using a fixed circular field (diameter of $750 \mu \mathrm{m}$ ) placed in the center of the brainstem medulla [including the area postrema (AP)]. Changes in expression were expressed as integrated density (as defined by Wang and McGinty, 1995), which is the product of area (highlighted pixels) $\times$ mean density. The graphs represent analysis from two to three sections per animal at the AP level.

Microphotography through a Leica (Nussloch, Germany) DMR microscope was done with a digital camera (CoolSnap; Photometrics, Tucson, AZ) and IPLab Spectrum software (Scanalytics, Fairfax, VA). All images were processed in Adobe Photoshop (Adobe Systems, San Jose, CA) using the Levels, Filter, and Contrast features to optimize them.

Statistical analysis. Group means were analyzed by ANOVA using Prism (GraphPad, San Diego, CA). Appropriate post hoc tests were used to determine significant differences between groups. 

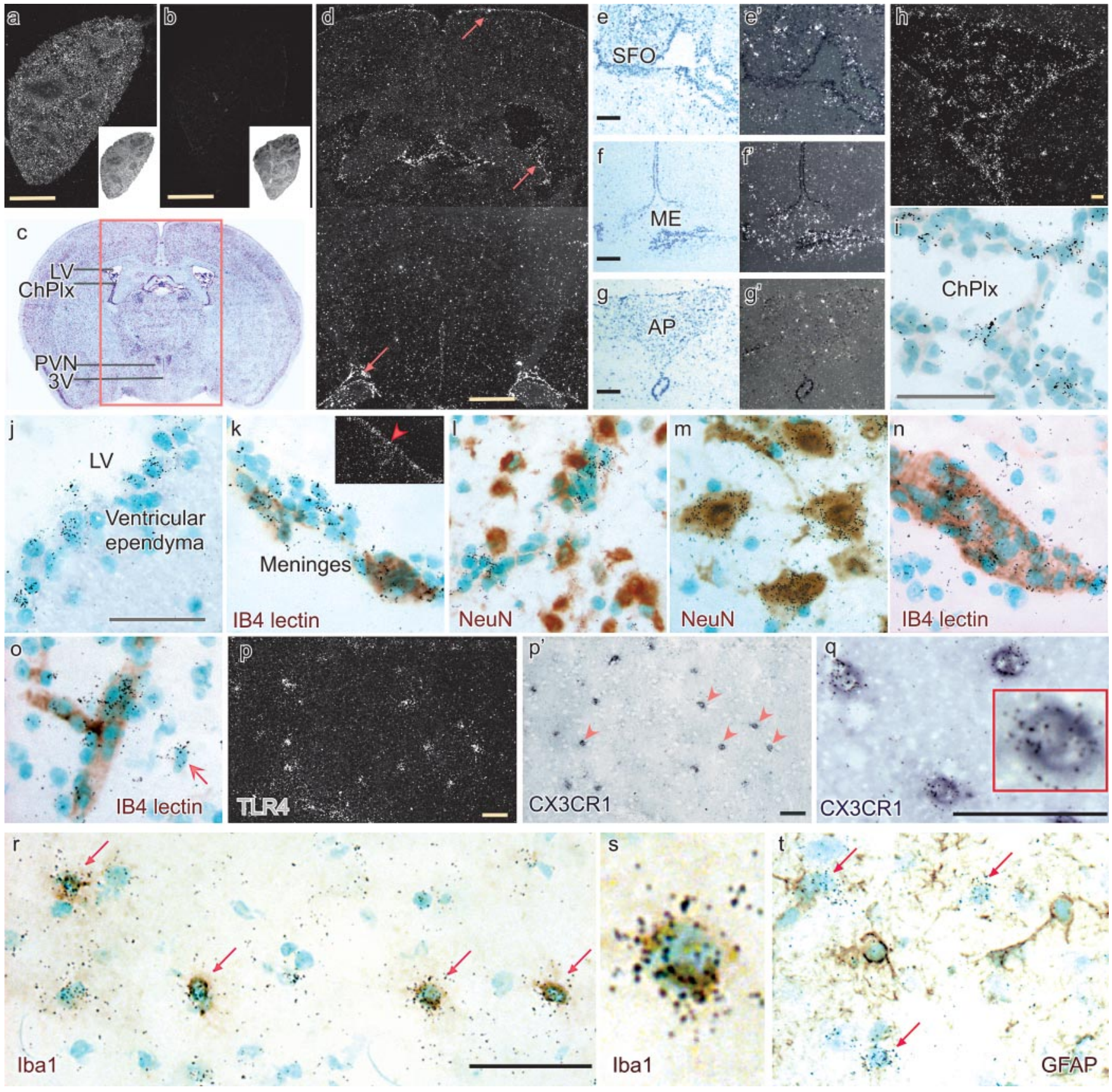

IB4 lectin

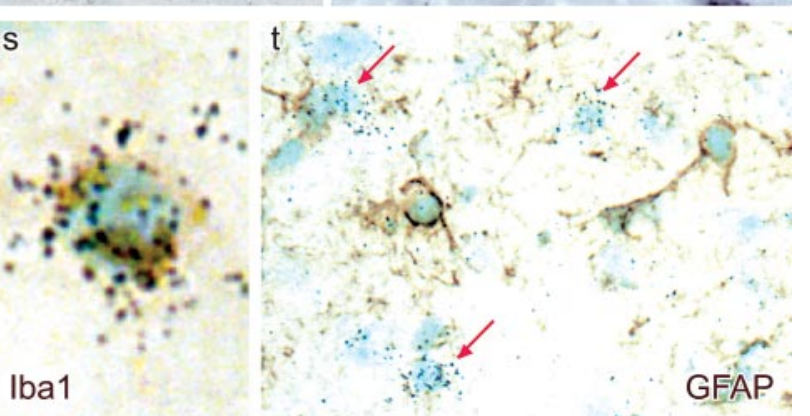

Figure 1. Distribution of TLR4 transcripts in the mouse brain. A full-length ${ }^{35} \mathrm{~S}$-labeled mouse TLR4 antisense riboprobe was hybridized to tissue sections singly ( $a-j$ ) or in combination with antibodies $(k-0)$ or a nonradioactive riboprobe $(p-q)$. Dark-field image shows TLR4 mRNA expression in a spleen section from $\mathrm{C3H} / \mathrm{HeOUJ}$ mice $(a)$ and not in $\mathrm{C57BL} / 10 \mathrm{ScC} r$ spleen $(b)$. Insets are bright-field images. Dark-field image ( $d$ ) corresponds to the brain area outlined in a Nissl-stained section ( $c$ ). Circumventricular organs, SF0 $(e)$, ME $(f)$, and AP $(g)$ show TLR4 expression when viewed in dark field $\left(e^{\prime}-g^{\prime}\right)$. Similarly, cells in choroid plexus $(h, i)$, ventricular ependyma $(j)$, and meninges $(k)$ express TLR4 mRNA. Hybridization with radioactive TLR4 cRNA was followed by immunohistochemistry with NeuN $(I, m)$ or a vascular marker isolectin B4 $(k, n, 0)$. Inset $(k)$ shows a low-power dark-field view of TLR4 mRNA in the meninges over cortex (arrowhead). Radioactive TLR4 mRNA (silver grains in the dark-field image, $p$ ) overlapped with a nonradioactive CX3CR1 label (purple cells in $p^{\prime}$ ). Magnified bright-field view ( $q$ ) and inset showing one double-labeled cell. TLR4 mRNA also colocalized with Iba1-positive microglial cells $\left(r\right.$, magnified in $\left.r^{\prime}\right)$, but not with GFAP-labeled astrocytes ( $s$ ). LV, Lateral ventricle; ChPlx, choroid plexus; PVN, paraventricular nucleus; $3 V$, third ventricle. Scale bars: $a, b, d, 1 \mathrm{~mm} ; e-h, 0.5 \mathrm{~mm} ; i-0,0.05 \mathrm{~mm} ; p, q, r, 0.05 \mathrm{~mm}$.

\section{Results}

TLR4 mRNA is widely expressed in the mouse CNS

The anatomical distribution of TLR4 mRNA was examined in mouse spleen and brain sections by in situ hybridization using an antisense riboprobe described in Materials and Methods. Spleen sections from wild-type $\mathrm{C} 3 \mathrm{H} / \mathrm{HeOuJ}$ (Fig. 1a) and C57BL/10SnJ (data not shown) mice showed robust hybridization in macrophage-rich areas of the red pulp and in cells surrounding the white pulp. Absence of hybridization to spleen sections from C57BL/10ScCr mice that have a genomic deletion of the TLR4 locus (Fig. $1 b$ ) showed probe specificity.

In the CNS, TLR4 mRNA expression was widespread across the brain parenchyma (Fig. 1d), with maximal expression in the choroid plexus and meninges (Fig. $1 d$, arrows). In CVOs like the subfornical organ (SFO) (Fig. 1e'), median eminence (ME) (Fig. $1 f^{\prime}$ ), and AP (Fig. $\left.1 g^{\prime}\right)$, TLR4 mRNA appeared in isolated cells 
and around fenestrated capillaries. We also observed TLR4 mRNA in choroid plexus cells (Fig. $1 h, i$ ), and in the ventricular ependyma (Fig. 1j). In meningeal tissue (Fig. 1k), the mRNA was associated with both blood vessels (marked by IB4 lectin staining) and nonvascular cells. The widespread distribution of TLR4 mRNA prompted us to examine whether it was expressed in neuronal cells. Lack of colocalization with an antibody against the neuronal marker NeuN confirmed that TLR4 was primarily in non-neuronal cells (Fig. 1l). However, some neurons, including the gigantocellular neurons of the medullary reticular formation, did express TLR4 mRNA (Fig. $1 \mathrm{~m}$ ). Within the brain parenchyma, hybridization was observed in cells lining large-diameter vessels (Fig. 1n,o, demarcated in these images by staining with IB4 lectin) and rarely in small vessels and capillaries. As seen in Figure 10 (arrow), individual parenchymal cells, distant from vessels, also expressed TLR4 transcripts.

Previous studies have reported CX3CR1 as a marker of microglia and astrocytes in the rodent brain. Using dual-label in situ hybridization, we demonstrated extensive overlap between TLR4 and CX3CR1 mRNA, confirming that parenchymal glial cells express TLR4 (Fig. $1 p^{\prime}$, arrowheads), seen also at high magnification (Fig. 1q). To further specify TLR4 mRNA expression in parenchymal cells, we combined in situ hybridization with immunohistochemistry for the microglia-macrophage marker Ibal or the astrocytic marker GFAP. TLR4-positive parenchymal cells expressed Ibal (Fig. 1r,s) but not GFAP (Fig. 1t). TLR4 expression in cells of the CNS parenchyma raises the possibility that LPS can be directly recognized to initiate inflammation in the CNS.
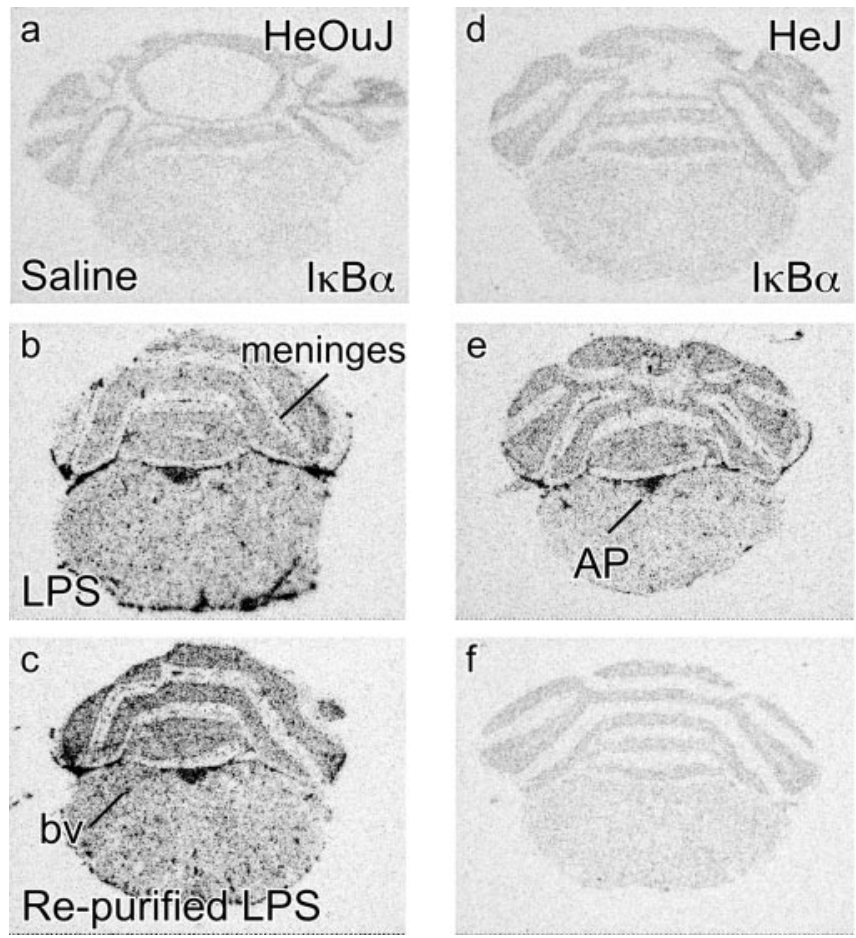

Figure 2. Effect of LPS repurification on gene transcription in TLR4 mutant mice. Film autoradiographs show induction of $\mathrm{I} \kappa \mathrm{B} \alpha(a-f)$ in brainstem sections of mice $2 \mathrm{~h}$ after LPS administration. $\mathrm{C} 3 \mathrm{H} / \mathrm{HeOuJ}(a-c)$ or $\mathrm{C} 3 \mathrm{H} / \mathrm{HeJ}$ mice $(d-f)$ were injected intraperitoneally with saline $(a, d)$; LPS serotype 055:B5 $(b, e)$ or repurified LPS $(c, f)$. bv, blood vessel. a

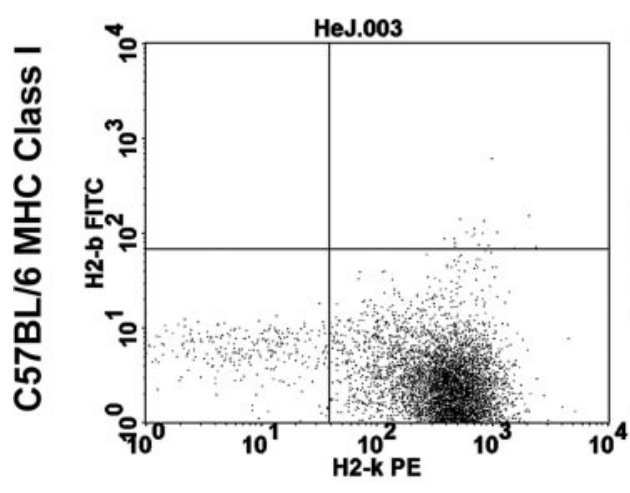

b

\section{HeJ MHC Class I}

Figure 3. Hematopoietic reconstitution replaces $>95 \%$ of resident $C D 45^{+}$cells. We assessed the efficiency of hematopoietic chimerism in our experimental protocol by making MHC class I mismatched chimeras. Bone marrow from B6 mice (MHC haplotype b) or HeOuJ mice (MHC haplotype k) were transferred into irradiated HeJ mice (MHC haplotype k). Two months after reconstitution, spleens were isolated from chimeric mice. Single-cell suspensions from spleen were labeled with antibodies to CD45 as well as antibodies specific to the MHC Class I molecules $(\mathrm{H}-2 \mathrm{~K})$ of the $\mathrm{k}\left(\mathrm{H}-2 \mathrm{~K}^{\mathrm{k}}\right)$ or b $\left(\mathrm{H}-2 \mathrm{~K}^{\mathrm{b}}\right)$ haplotype. Staining was analyzed on a BD FACScalibur. Plots show MHC staining observed on cells that are $\mathrm{CD} 45^{+}$(gated). In the case of mice receiving HeOuJ bone marrow (a), $\sim 96 \%$ of the $\mathrm{CD} 45^{+}$cells were of the HeOuJ origin. In the case of mice receiving the B6 bone marrow $(b), \sim 91 \%$ of the cells stained for the $\mathrm{B} 6$ Class I molecule. Although $\sim 2 \%$ of the cells fell within the bottom right quadrant, suggesting a staining by the k haplotype, there was no distinct population corresponding to the MHC-expressing cells (compare bottom right quadrants in $a$ ). This allows us to conclude that our protocols were successful in generating at least $90-95 \%$ donor-derived hematopoietic chimerism with no significant contribution from the recipient toward this compartment. Although this analysis was done using MHC-mismatched chimeras involving a protocol for depletion of resident NK cells before reconstitution, those WT and HeJ mice that were used to construct the experimental chimeras did not involve a mismatch at the MHC locus.

\section{The HeJ CNS is refractory to inflammation induced by repurified LPS}

Inflammatory agonists induce a variety of transcripts including $\mathrm{I} \kappa \mathrm{B} \alpha, \mathrm{CD} 14, \mathrm{IL}-1 \beta$, TNF $\alpha$, TLR2, and monocyte chemoattractant protein-1 (Quan et al., 1997; Laflamme et al., 2003) in the CNS. We first tested the consequence of TLR4 dysfunction on CNS inflammatory responses by measuring the upregulation of $\mathrm{I} \kappa \mathrm{B} \alpha$ mRNA after peripheral administration of LPS. Two hours after challenge, I $\kappa \mathrm{B} \alpha$ mRNA was upregulated in $\mathrm{HeOuJ}$ mice (WT for TLR4) several-fold above salineinjected controls (Fig. 2a,b). Predominant induction was observed around blood vessels and in CVOs like the AP. Comparable results were also observed for IL-1 $\beta$ and CD14 mRNA (data not shown). Surprisingly, a qualitatively similar pattern of inflammatory upregulation was reproducibly $(n=6)$ seen in HeJ mice brains (Fig. 2e).

Manthey et al. (1994) have previously reported contaminants in commercial LPS that could induce inflammation in a TLR4-independent manner. We therefore purified the commercial LPS preparation as described in Materials and Methods. Repurification completely eliminated responses to LPS administration in the HeJ brain (Fig. 2f); expression patterns of I $\kappa \mathrm{B} \alpha$ 
mRNA in $\mathrm{HeJ}$ mice were indistinguishable from saline controls. Purification did not alter mRNA expression in WT mice compared with the commercial LPS (Fig. 2b,c). The repurified preparation of LPS was therefore used in all subsequent experiments.

\section{WT hematopoietic cells restore} peripheral inflammation in $\mathrm{HeJ}$ mice The clearly unresponsive phenotype of $\mathrm{HeJ}$ mice allowed us to further evaluate the importance of LPS-TLR4 interactions on distinct cellular lineages in shaping the immune response to LPS. We first examined the ability of wild-type TLR4-expressing hematopoietic cells to overcome the HeJ deficiency in peripheral inflammatory responses. We made four combinations of radiation chimeras by hematopoietic reconstitution of $\mathrm{HeJ}$ or WT recipient mice with bone marrow of either type. The chimeras are summarized as $\mathrm{WT} \rightarrow \mathrm{WT}$, HeJ $\rightarrow \mathrm{HeJ}, \mathrm{WT} \rightarrow \mathrm{HeJ}$, and $\mathrm{HeJ} \rightarrow \mathrm{WT}$. Twelve weeks after the transfer, $>95 \%$ of the splenic hematopoietic cells were donor-derived (Fig. 3). The chimeric mice were then challenged with an intraperitoneal injection of $1 \mathrm{mg} / \mathrm{kg}$ repurified LPS.

Reconstitution of HeJ mice with WT $\mathrm{BM}$ resulted in the induction of TNF $\alpha$, IL$1 \beta$, IL-12p70, and IFN $\gamma$ in the blood (Fig. $4 a-d)$, closely resembling the response of $\mathrm{WT} \rightarrow$ WT control chimeric mice (Fig. 4). In both groups, TNF $\alpha$ (Fig. $4 a$ ) was induced early $(2 \mathrm{~h})$ and was undetectable by 6 h. IL-1 $\beta$ (Fig. $4 b$ ) and IL-12p70 (Fig. $4 c$ ) could be detected as early as $2 \mathrm{~h}$, and secretion continued at $6 \mathrm{~h}$. IFN $\gamma$ required at least $6 \mathrm{~h}$ before significant levels could be detected (Fig. $4 d$ ).

In contrast, transplanting $\mathrm{HeJ} \mathrm{BM}$ into WT recipients resulted in severely attenuated serum cytokine levels in the host. Parallel findings were obtained for measures of TNF $\alpha$ mRNA in the spleens of chimeric mice (Fig. $4 e-h$ ). These observations clearly demonstrate a requirement for hematopoietic TLR4 function in eliciting peripheral inflammation after systemic LPS.

\section{Early inflammatory responses in the $\mathrm{CNS}$ of $\mathrm{HeJ}$ mice are partially restored by WT hematopoietic cells}

The chimeric mice with lineage-restricted TLR4 function were then used to examine the CNS inflammatory response during endotoxemia. TNF $\alpha$ and $\mathrm{I} \kappa \mathrm{B} \alpha$ transcripts were highly upregulated in the brains of WT $\rightarrow$ WT chimeric mice $2 \mathrm{~h}$ after intraperitoneal LPS (Fig. 5b,f). This induction was almost nonexistent in $\mathrm{HeJ} \rightarrow \mathrm{HeJ}$ mice (Fig. 5a,e). Quantitatively, TNF $\alpha$ mRNA levels in $\mathrm{WT} \rightarrow \mathrm{WT}$ mice exceeded those of $\mathrm{HeJ} \rightarrow \mathrm{HeJ}$ by almost 10 -fold $(p<0.001$ ) (Fig. 5i). In WT $\rightarrow \mathrm{HeJ}$ chimeric mice (Fig. $5 c, g)$, mRNA levels of TNF $\alpha$ and $\mathrm{I} \kappa \mathrm{B} \alpha$ were significantly higher (3.5- and 5.3-fold) than in $\mathrm{HeJ} \rightarrow \mathrm{HeJ}$ mice but were less than the $\mathrm{WT} \rightarrow$ WT levels (Fig. 5b,f). Similarly, lower levels of TNF $\alpha$ (Fig. $5 d$ ) and $\mathrm{I} \kappa \mathrm{B} \alpha$ (Fig. 5h) transcripts were induced in the HeJ $\rightarrow$ WT chimeras, relative to WT $\rightarrow$ WT mice. Thus, although TLR4 expression in the BM lineage was sufficient to mediate complete LPS responsiveness in the periphery, this was rather ineffective in recovering the early response within the CNS.
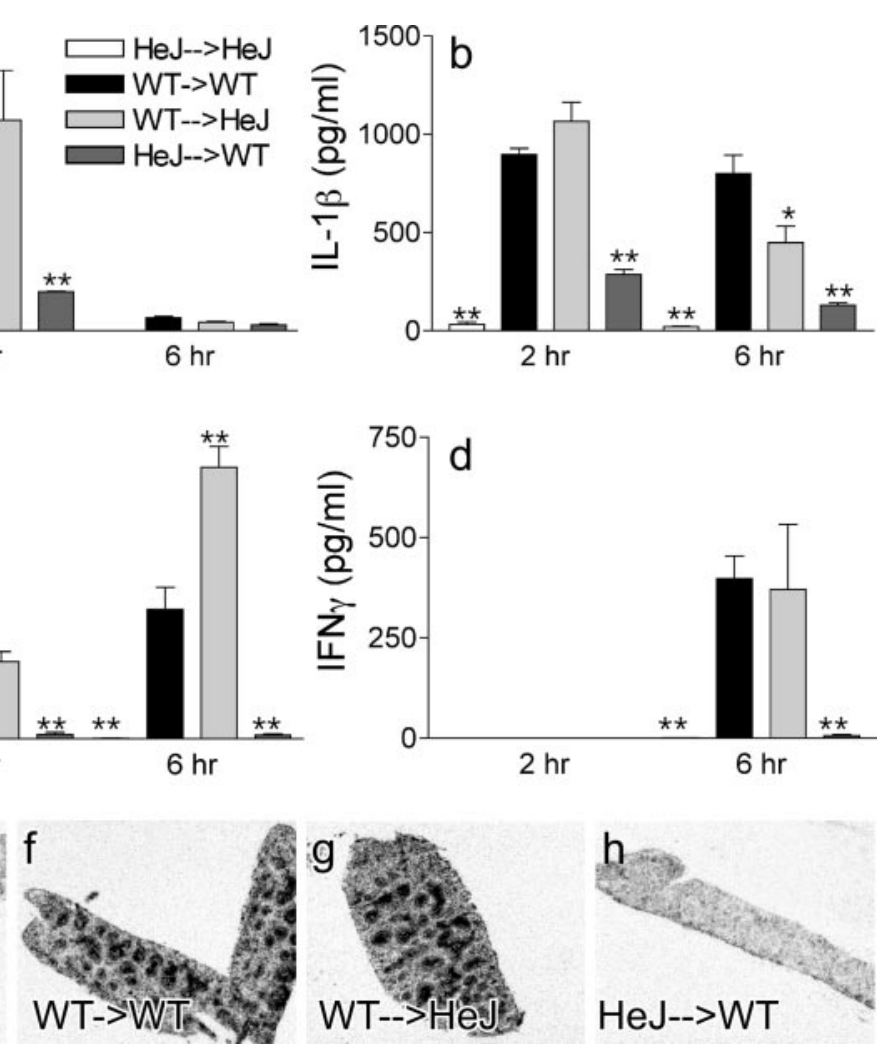

HeJ-->WT

Figure 4. Induction of peripheral cytokines in TLR4 chimeric mice. Mice were challenged with repurified LPS (1 mg/kg, i.p.), trol WT $\rightarrow$ WT chimeric mice after one-way ANOVA. ${ }^{*} p<0.05 ;{ }^{* *} p<0.001$. Autoradiographic images show TNF $\alpha$ mRNA in the spleen of chimeric mice: HeJ $\rightarrow$ HeJ $(e)$, WT $\rightarrow$ WT $(f)$, WT $\rightarrow$ HeJ $(g)$, and HeJ $\rightarrow$ WT $(h) 2$ h after LPS administration.

\section{Late inflammatory response in the CNS requires parenchymal TLR4 signaling}

We then examined whether continued inflammatory sequelas in the brain required hematopoietic recognition of LPS. Six hours after LPS challenge, brain sections of WT $\rightarrow$ WT chimeric mice displayed high levels of $\mathrm{I} \kappa \mathrm{B} \alpha$, IL-1 $\beta$, TLR2, and CD14 mRNA expression. For all four mRNA species, no detectable transcriptional induction was observed in $\mathrm{HeJ} \rightarrow \mathrm{HeJ}$ chimeric animals (Fig. 6). A dense hybridization to the $\mathrm{I} \kappa \mathrm{B} \alpha$ probe was observed in the AP and vascular structures in the parenchyma and meninges of the WT $\rightarrow$ WT chimeric group (Fig. $6 b$ ). IL-1 $\beta$ and TLR2 (Fig. $6 f, j)$ were intensely induced in scattered cells throughout the brain. TLR2 was also highly induced in the AP, and a strong parenchymal labeling was observed. CD14 mRNA expression was high in meninges and vascular-associated and parenchymal cells (Fig. 6n).

In comparison, the WT $\rightarrow \mathrm{HeJ}$ chimeras (Fig. $6 c, g, k, o$ ) showed a significantly reduced response at $6 \mathrm{~h}$, even although the levels were higher than in $\mathrm{HeJ} \rightarrow \mathrm{HeJ}$ mice. I $\kappa \mathrm{B} \alpha$ transcription was reduced at least three-fold (Fig. $6 q$ ), IL-1 $\beta$ and TLR 2 by more than twofold (Fig. 6r,s), and CD14 by more than threefold (Fig. $6 t)$ relative to $\mathrm{WT} \rightarrow \mathrm{WT}$.

In surprising contrast to the $2 \mathrm{~h}$ pattern, however, WT recipients of HeJ BM (Fig. $6 d, h, l, p$ ) showed mRNA induction levels and patterns comparable with the WT $\rightarrow$ WT response at $6 \mathrm{~h}$. Levels of $\mathrm{I} \kappa \mathrm{B} \alpha$ mRNA were reduced in $\mathrm{HeJ} \rightarrow \mathrm{WT}$ by $\sim 10 \%$ relative to WT $(p<0.05$ ) (Fig. $6 q$ ), but IL-1 $\beta$ (Fig. $6 r$ ), TLR2 (Fig. 6s), and CD14 (Fig. 6t) mRNA levels were not significantly 

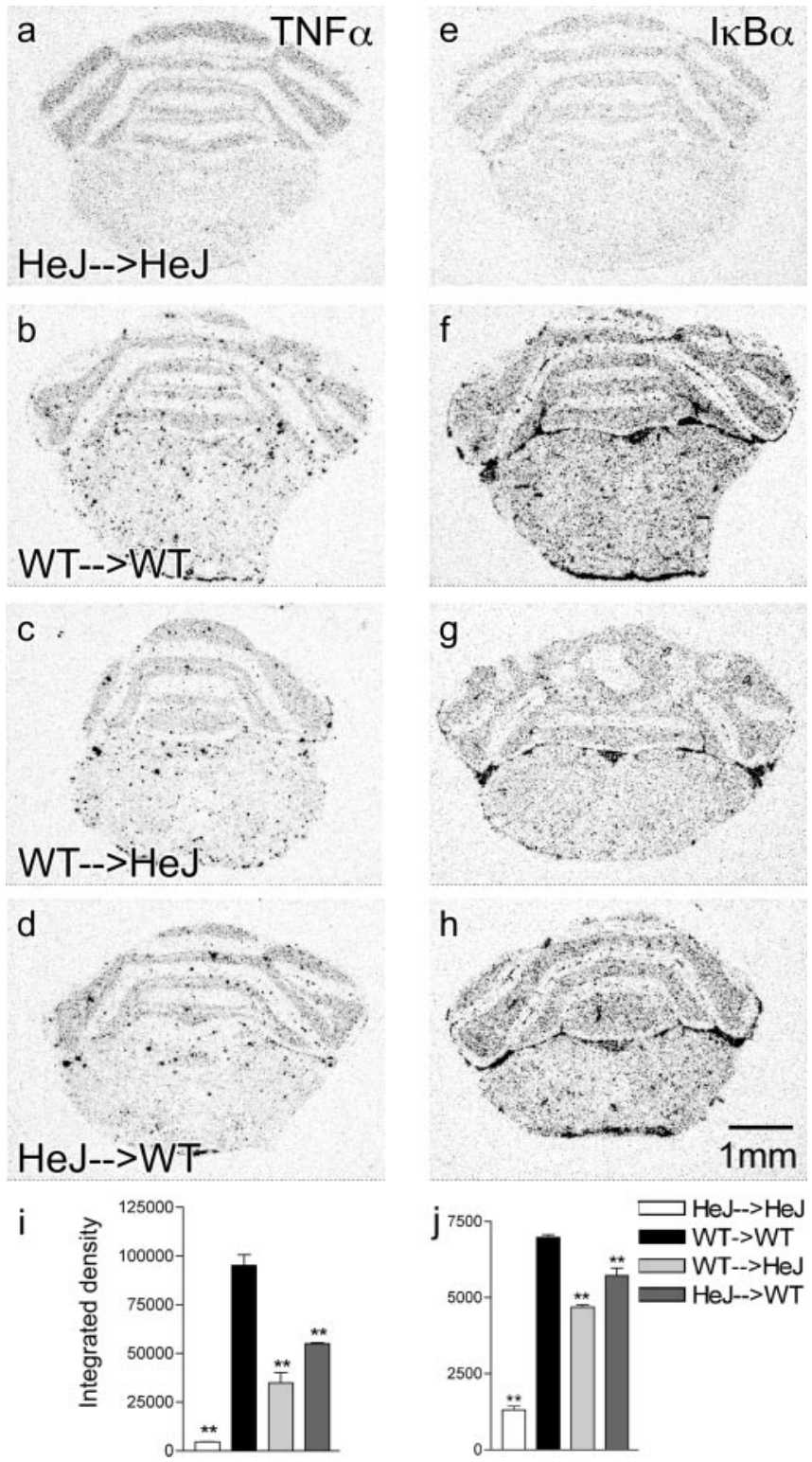

Figure 5. Early inflammatory response to LPS in CNS of chimeric mice. Film autoradiographs $(a-h)$ show mRNA induction in brains of mice killed $2 \mathrm{~h}$ after intraperitoneal LPS. TNF $\alpha(a-d)$ and $\mathrm{I}_{\kappa} \mathrm{B} \alpha(e-h)$ mRNA in brainstem sections of the chimeric mice: HeJ $\rightarrow \mathrm{HeJ}(a, e), \mathrm{WT} \rightarrow$ WT $(b, f)$, WT $\rightarrow$ HeJ $(c, g)$, and HeJ $\rightarrow$ WT $(d, h)$. Induction of TNF $\alpha(i)$ and $\mathrm{I}_{\kappa} \mathrm{B} \alpha(j)$ mRNA in chimeric groups (details in key) were quantified by densitometry of film images. Data representing mean integrated density \pm SEM for each mRNA were obtained from three or four mice per group. One-way ANOVA was followed by appropriate post hoc analysis to compare chimeric group means against the control WT $\rightarrow$ WT group. ${ }^{* *} p<0.001$.

different from those in WT $\rightarrow$ WT mice. Also, in WT $\rightarrow$ WT and $\mathrm{HeJ} \rightarrow \mathrm{WT}$ chimeras, mRNA levels of CD14, TLR2, and IL-1 $\beta$ increased at $6 \mathrm{~h}$ relative to $2 \mathrm{~h}$, whereas in WT $\rightarrow \mathrm{HeJ}$ mice they decreased (data not shown). Therefore, the early and late CNS responses to peripheral endotoxin depend on TLR4 function in distinct cellular compartments. Both hematopoietic and resident cells participate in the inductive phase of the response that later evolves into a predominantly resident cell-driven process, although circulating cytokines or bone marrow-derived cells within the brain may be responsible for some of the inflammatory response observed at blood-brain barrier-associated sites.

An interesting feature emerged in the qualitative pattern of gene expression in different chimeric animals, based on micro- scopic examination of emulsion-coated sections. Although the overall cytokine mRNA levels were reduced in WT $\rightarrow \mathrm{HeJ}$ chimeras at $6 \mathrm{~h}$, positive signal was still evident in barrier-associated cells. I $\kappa \mathrm{B} \alpha$, IL-1 $\beta$, TLR2, and CD14 mRNAs were expressed in meningeal cells and blood vessels (Fig. $6 c, g, k, o$ ). This was similar to the anatomical distribution of $\mathrm{I} \kappa \mathrm{B} \alpha$ (Fig. $5 g$ ), TLR2, and CD14 (data not shown) mRNAs at $2 \mathrm{~h}$ after LPS, suggesting that the $6 \mathrm{~h}$ pattern is a continuation of the previous response elicited by TLR4-positive bone marrow-derived cells in WT $\rightarrow \mathrm{HeJ}$ mice. Likewise, in $\mathrm{HeJ} \rightarrow$ WT chimeras, $\mathrm{I} \kappa \mathrm{B} \alpha$, TLR2, and CD14 mRNAs were expressed in meninges, blood vessels, and circumventricular organs at $2 \mathrm{~h}$ (Fig. $5 h$ ) (data not shown) and $6 \mathrm{~h}$ (Fig. $6 d, h, l, p)$. An additional feature of the $\mathrm{HeJ} \rightarrow$ WT chimeras, not evident in $\mathrm{WT} \rightarrow \mathrm{HeJ}$, was a spread of inflammatory transcript expression to include parenchymal cells at $6 \mathrm{~h}$ after LPS. Although a detailed quantitative analysis of labeled cells in barrier versus parenchymal regions was not performed in this study, CD14, TLR2, and IL- $1 \beta$ mRNA-positive cells were observed away from barrier-associated areas in $\mathrm{HeJ} \rightarrow \mathrm{WT}$ but not WT $\rightarrow \mathrm{HeJ}$ mice (Fig. 6). Finally, some mRNAs had unique induction patterns. Transcripts for $\mathrm{I} \kappa \mathrm{B} \alpha$ at $6 \mathrm{~h}$ were more diffusely spread over the tissue in both chimeras than were the aforementioned markers, and transcripts for TNF $\alpha$ were localized to sparsely spaced barrier cells at $6 \mathrm{~h}$ in both groups.

\section{Nonhematopoietic TLR4 activation is responsible for a temporal rise in serum corticosterone levels}

Endotoxemia alters the physiology of the HPA axis in mammals and results in cortisol or corticosterone release from the adrenal medulla (Turnbull and Rivier, 1999). Using TLR4 chimeric mice, we tested whether the HPA axis response to LPS is affected by lineage restriction of TLR4 function. Serum was collected from chimeric mice 2 or $6 \mathrm{~h}$ after an intraperitoneal injection of purified LPS ( $1 \mathrm{mg} / \mathrm{kg})$, and corticosterone levels were measured by radioimmunoassay against murine corticosterone (Fig. 7 ). The most striking feature of the corticosterone profile in chimeric mice was a similarity in the response between WT $\rightarrow \mathrm{WT}$ and $\mathrm{HeJ}$ $\rightarrow$ WT groups at 2 and $6 \mathrm{~h}$ after LPS $(p>0.05)$. In comparison, corticosterone levels in $\mathrm{WT} \rightarrow \mathrm{HeJ}$ chimeras started out at a lower level than in WT $\rightarrow$ WT mice at $2 \mathrm{~h}$ (statistically insignificant), and were reduced to even lower levels by $6 \mathrm{~h}(p<0.001)$. The trend contrasted sharply with that seen in $\mathrm{HeJ} \rightarrow$ WT chimeric mice in which corticosterone levels rose from 2 to $6 \mathrm{~h}$, similar to the trend in WT $\rightarrow$ WT animals.

Interestingly, an inverse correlation was observed in the chimeric groups between levels of corticosterone and cytokines in serum. In the $\mathrm{WT} \rightarrow \mathrm{HeJ}$ chimeric group (showing reduced corticosterone levels at $6 \mathrm{~h}$ ) (Fig. 7), the $6 \mathrm{~h}$ increase in IL-12p70 concentration in blood far exceeded (Fig. 4c) that in WT $\rightarrow$ WT chimeras (with a higher glucocorticoid level at $6 \mathrm{~h}$ ).

In a separate experiment in $27 \mathrm{C} 3 \mathrm{H} / \mathrm{HeN}$ mice receiving LPS ( $1 \mathrm{mg} / \mathrm{kg}$, i.p.) or vehicle and surviving $0.5,2$, or $6 \mathrm{~h}$ after injection, densitometry of a region including the choroid plexus of the lateral ventricle showed that levels of TLR4 gene expression were unaltered by LPS treatment (data not shown).

\section{Discussion}

The CNS inflammatory sequelas that follow peripheral LPS administration have provided a model system to study the regulation of CNS-mediated components of an acute phase response to infection. The cellular compartments initiating various aspects of this process are, however, not clearly defined. Using a sensitive in situ hybridization technique, we demonstrate TLR4 mRNA expression in the mouse brain. The hitherto unreported detection 
of this transcript in parenchymal microglia in situ, in addition to epithelial and vascular-derived structures of the mouse CNS, raised the possibility that local endotoxin recognition occurs during the CNS inflammatory response to systemic LPS. We were then able to uncouple the cellular compartments mediating direct and indirect recognition of LPS in the brain using $\mathrm{BM}$ radiation chimeras between WT LPSresponsive mice and TLR4 mutant mice with a defect in endotoxin signaling. The importance of resident TLR4-bearing cells in the induction and especially maintenance of the inflammatory response in the CNS is underscored by two observations. First, we find that reconstitution of LPShyporesponsive HeJ mice with TLR4positive WT BM only partially restores LPS-mediated induction of proinflammatory transcripts in the recipient's CNS. Second, we find that TLR4 function in nonhematopoietic cells alone is sufficient for inflammatory progression in the CNS and corticosterone elevation in the serum of LPS-treated WT mice containing TLR4deficient peripheral immune cells. These findings provide direct evidence for LPS signaling in tissue-resident CNS cells as a mechanism for inflammatory transduction in the brain and increased HPA axis activity during endotoxemia.

Recognition of LPS critically requires cell-autonomous expression of TLR4. In the mouse brain, distribution of cells expressing TLR4 is very widespread, more extensive than previously reported for the rat brain (Laflamme and Rivest, 2001). Many TLR4-positive cells were observed along large brain vessels and within the choroid plexus, meninges, CVOs, and the ventricular ependymal lining (Fig. 1). The exact identity of TLR4-bearing cells at these locations is unresolved and, similar to peripheral tissues, may include myeloid (Rehli et al., 2000; Oshikawa and Sugiyama, 2003), endothelial (Faure et al., 2001; Coats et al., 2003), or epithelial (Cario and Podolsky, 2000; Song et al., 2001) lineages. One novel finding from the highresolution analysis of TLR4 distribution was its presence in parenchymal microglial cells but not astrocytes in the brain parenchyma.

We cannot say that all of the TLR4-positive cells in the brain are host-derived in the chimeras. Much recent work has shown the possibility that pluripotent BM stem cells enter the brain and differentiate into various neural and non-neural cell types in adult, unperturbed animals. Cells of hematopoietic origin in the BM chimera model have been tracked in a recent definitive study (Vallieres and Sawchenko, 2003), which showed that 3 months after irradiation and BM transplantation in normal adult mice, a small fraction of marked BM cells differentiate into perivascular cells (Vallieres and Sawchenko, 2003). Most of the donor-derived cells were located outside the blood-brain barrier, i.e., in the
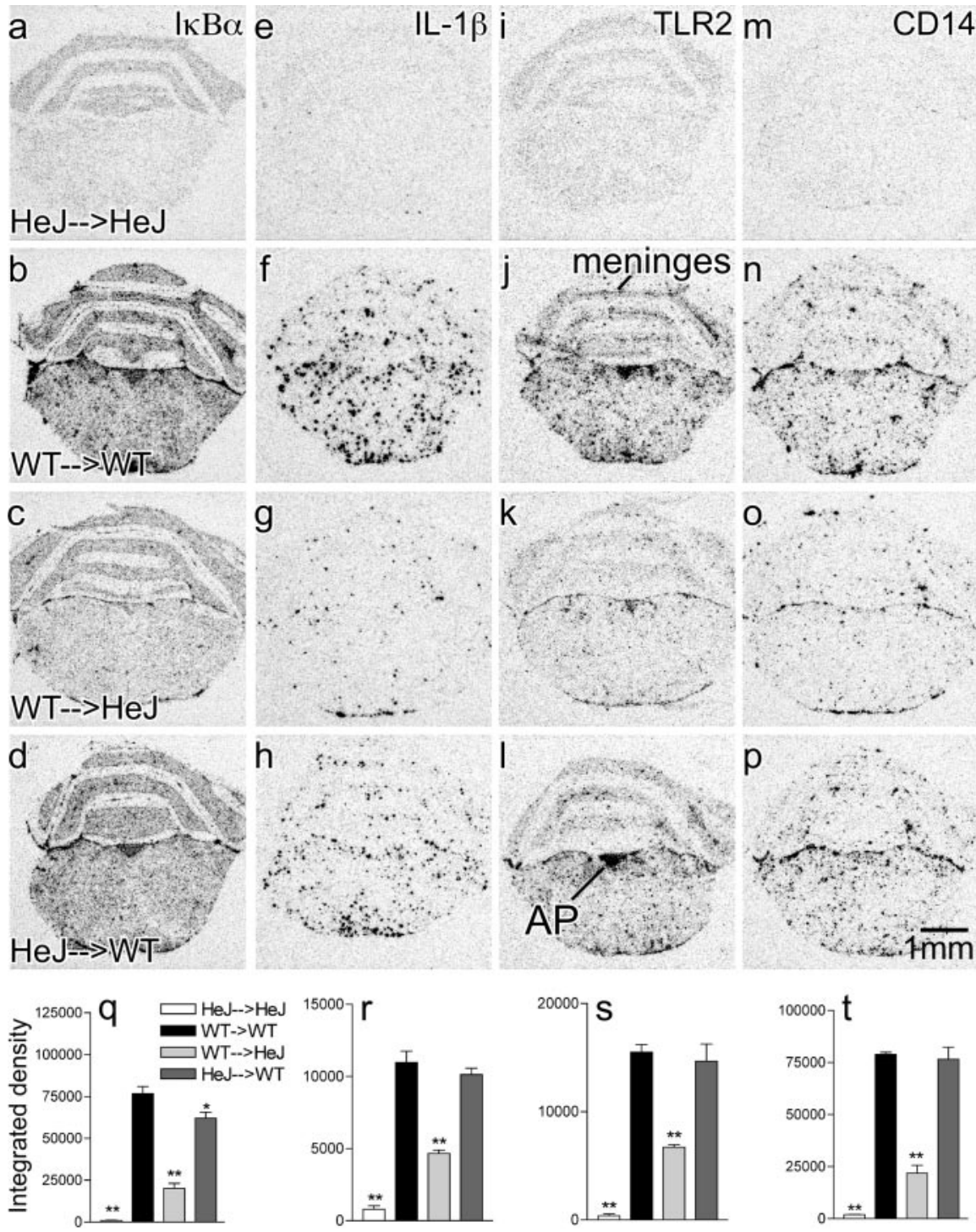

Figure 6. Late inflammatory response to LPS in CNS of chimeric mice. Representative film autoradiographs $(a-p)$ show proinflammatory mRNA induction in brains of mice killed $6 \mathrm{~h}$ after intraperitoneal LPS. I $\kappa \mathrm{B}, \mathrm{IL}-1 \beta, \mathrm{TLR} 2(i-l)$, and CD14 ( $m-p)$ mRNAs were detected by radioactive in situ hybridization on brainstem sections from chimeric mice: HeJ $\rightarrow$ HeJ $(a, e, i, m)$, WT $\rightarrow$ TLR2 $(s)$, and CD14 $(t)$ mRNA levels are plotted as mean integrated density \pm SEM from three or four mice per chimeric class. Individual group means were compared against the control WT $\rightarrow$ WT group, and statistical significance was established by one-way ANOVA and appropriate post hoc tests. ${ }^{*} p<0.05$; ${ }^{* *} p<0.001$.

choroid plexus and in the circumventricular organs, and those in the brain were associated with blood vessels and were identified as perivascular microglia by the criteria of morphology and positive immunostaining with Ibal antibody (Vallieres and Sawchenko, 2003). Previous claims that BM cells in normal mice enter the brain parenchyma and differentiate into neural cell types was not supported in this and another careful study (Wehner et al., 2003). However, it is apparent that a small fraction of perivascular cells in restricted locations in the CNS are BM derived in chimeric mice 3 months after BM transplantation (see below).

The purpose of generating chimeric mice that lack TLR4 receptor function in hematopoietic or resident cells was to ask whether there is tissue-specific recognition of circulating LPS in an in vivo endotoxemia model and, if so, which compartment drives the CNS response. To our knowledge, we are first able to 


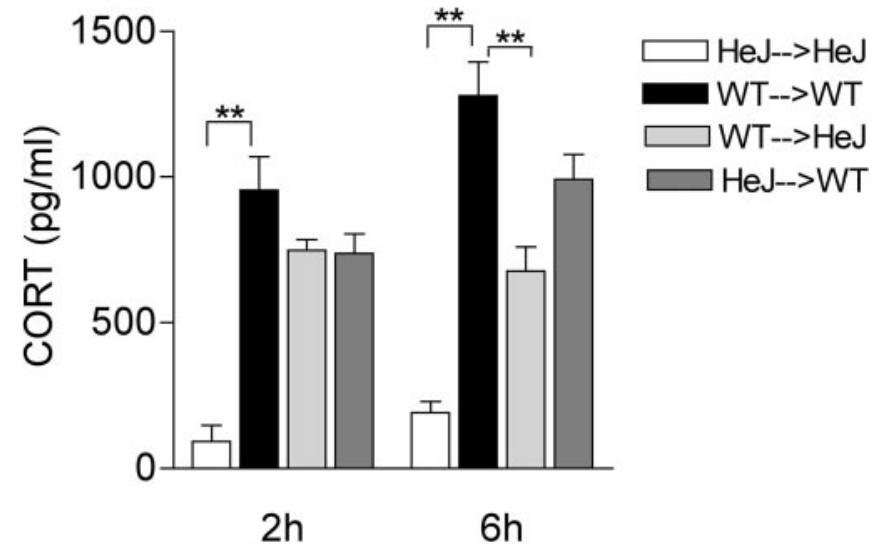

Figure 7. Corticosterone (CORT) levels in serum from LPS-treated chimeric mice. Corticosterone was detected in serum from chimeric mice 2 or $6 \mathrm{~h}$ after an intraperitoneal injection of purified LPS (1 mg/kg), using a radioimmunoassay kit directed against murine corticosterone. Each bar represents mean \pm SEM of values from three or four independent animals per group. Significant differences were established by two-way ANOVA, and post hoc tests were used to compare chimeric group means against the WT $\rightarrow$ WT control. ${ }^{* *} p<0.001$.

unequivocally evaluate TLR4 dependence of the LPS response in vivo by repurifying LPS and eliminating TLR4-independent immunostimulatory activity imparted by contaminants in commercial preparations. Our results with chimeric mice suggest that LPS-TLR4 interactions in distinct sets of cells drive the temporal pattern of CNS inflammation. At the onset of the CNS response, measured by transcriptional activation of $\mathrm{I} \kappa \mathrm{B} \alpha$ and $\mathrm{TNF} \alpha$ at $2 \mathrm{~h}$ after LPS administration, TLR4 function is required in both BMderived cells and resident tissue cells. BM-derived cells in this context include circulating leukocytes and perivascular cells along the meninges or around vascular structures as described previously (Vallieres and Sawchenko, 2003). Early TLR4 activation on hematopoietic or resident cells alone $(\mathrm{WT} \rightarrow \mathrm{HeJ}$ and $\mathrm{HeJ}$ $\rightarrow$ WT mice) partially contributes to the WT response seen at $2 \mathrm{~h}$ in WT $\rightarrow$ WT CNS (Fig. 5). However, hematopoietic TLR4 activation leads to either limited production of secondary mediators or limited access of these factors to sites responsible for propagation of CNS inflammation. As a result, at $6 \mathrm{~h}$ after LPS administration, WT $\rightarrow \mathrm{HeJ}$ chimeric mice exhibit a drastically reduced response compared with the WT $\rightarrow$ WT pattern (Fig. 6). In contrast, TLR4 signaling in tissue-resident cells (HeJ $\rightarrow$ WT chimera) allows elaboration of a $6 \mathrm{~h}$ inflammatory response that is almost indistinguishable from that in WT $\rightarrow$ WT mice. The relevant resident cells bearing TLR4 may include parenchymal microglia that are not derived from BM progenitors in vivo (Vallieres and Sawchenko, 2003; Wehner et al., 2003) as well as endothelial, choroidal epithelial, meningeal, fibroblast, or ependymal cells, which also cannot be replaced by BM cells.

In this study, we were able to unequivocally evaluate TLR4 dependence of the LPS response in vivo by repurifying LPS and eliminating TLR4-independent immunostimulatory activity imparted by contaminants in commercial preparations. The dissociation of inflammatory responses between WT $\rightarrow \mathrm{HeJ}$ and $\mathrm{HeJ}$ $\rightarrow$ WT chimeric groups suggests the following possibilities. LPS may affect radiation-resistant cellular subsets at the same time as BM-derived lineages, and only the former can sustain an inflammatory milieu by virtue of their anatomical location or selective response profile. Alternatively, LPS might gain access to additional resident cells after passing the blood-brain or blood-CSF barriers over time, to drive the sustained CNS inflammation seen at $6 \mathrm{~h}$. An inflammatory response in blood-brain barrier sites is detected at $6 \mathrm{~h}$ in $\mathrm{WT} \rightarrow \mathrm{HeJ}$ mice. This may be elicited by bone marrow-derived cells that have repopulated vascular sites in the brain, or by circulating cytokines in blood. Gene expression in barrier versus parenchymal cells may qualitatively and quantitatively differ somewhat among the mRNAs analyzed, but TLR4 expression on hematopoietic cells in this chimera is responsible for transcripts induced in the barrier cells.

It is noteworthy that the response at $6 \mathrm{~h}$ in $\mathrm{HeJ} \rightarrow$ WT CNS is also independent of circulating cytokines. The rise of serum cytokines is almost entirely eliminated in $\mathrm{HeJ} \rightarrow \mathrm{WT}$ chimeric mice and seems to depend exclusively on TLR4 function in hematopoietic cells (Fig. 4). Despite this, proinflammatory gene transcription in the CNS proceeds unabated in this group of animals. Interestingly, serum levels of corticosterone in the $\mathrm{HeJ} \rightarrow \mathrm{WT}$ group were elevated at the same level as WT $\rightarrow$ WT at $6 \mathrm{~h}$, whereas they were significantly reduced in the WT $\rightarrow \mathrm{HeJ}$ group (Fig. 7), supporting the important role of the CNS parenchyma in elaborating a physiological response (HPA axis activation). Whether nonhematopoietic TLR4 signaling selectively or uniformly affects all levels of the HPA axis will be addressed in future studies.

A functional consequence of the increased HPA axis activity during endotoxemia is suggested by the inverse correlation between glucocorticoid levels and the rise in IL-12p70 levels in serum at $6 \mathrm{~h}$ after LPS. In the WT $\rightarrow \mathrm{HeJ}$ chimeric group (showing reduced corticosterone levels at 6 h relative to $\mathrm{WT} \rightarrow \mathrm{WT}$ ) (Fig. 7 ), the $6 \mathrm{~h}$ increase in IL-12p70 concentration in blood far exceeds (Fig. 3c) that in WT $\rightarrow$ WT chimeras. The other cytokines at their peak also show higher (but not significant) levels in the $\mathrm{WT} \rightarrow \mathrm{HeJ}$ group than in the WT $\rightarrow$ WT group (TNF and IL- $1 \beta$ at $2 \mathrm{~h}$ and IL-12p70 and IFN $\gamma$ at $6 \mathrm{~h}$ ). Thus, it is attractive to speculate that TLR4 activation on nonhematopoietic cells (absent in WT $\rightarrow$ HeJ chimeric mice) may contribute to the containment of excessive cytokine release in the periphery via modulation by glucocorticoids or other unexplored mechanisms.

We cannot rule out the contribution of serum cytokines in CNS immune activation or the glucocorticoid surge at $2 \mathrm{~h}$ after LPS because the responses are partial in both $\mathrm{HeJ} \rightarrow \mathrm{WT}$ and WT $\rightarrow \mathrm{HeJ}$ mice, compared with the WT $\rightarrow \mathrm{WT}$ group. It is possible, however, that CNS inflammation at $2 \mathrm{~h}$ in $\mathrm{WT} \rightarrow \mathrm{HeJ}$ mice arises from LPS-TLR4 interactions on perivascular hematopoietic cells that have migrated into the brain during reconstitution after irradiation. In that case, the $2 \mathrm{~h}$ pattern in these mice may also be independent of circulating cytokines and be driven by local TLR4 signaling on perivascular cells. Nevertheless, the chimeric TLR4 model establishes a complete dissociation between peripheral cytokines in serum and late inflammatory events within the brain as well as demonstrating a functional consequence of direct recognition of the endotoxin by CNS resident cells.

\section{References}

Adams DH, Hanson GR, Keefe KA (2000) Cocaine and methamphetamine differentially affect opioid peptide mRNA expression in the striatum. J Neurochem 75:2061-2070.

Berkenbosch F, van Oers J, del Rey A, Tilders F, Besedovsky H (1987) Corticotropin-releasing factor-producing neurons in the rat activated by interleukin-1. Science 238:524-526.

Breder CD, Saper CB (1996) Expression of inducible cyclooxygenase mRNA in the mouse brain after systemic administration of bacterial lipopolysaccharide. Brain Res 713:64-69.

Breder CD, Hazuka C, Ghayur T, Klug C, Huginin M, Yasuda K, Teng M, Saper CB (1994) Regional induction of tumor necrosis factor $\alpha$ expression in the mouse brain after systemic lipopolysaccharide administration. Proc Natl Acad Sci USA 91:11393-11397.

Bsibsi M, Ravid R, Gveric D, van Noort JM (2002) Broad expression of 
Toll-like receptors in the human central nervous system. J Neuropathol Exp Neurol 61:1013-1021.

Cario E, Podolsky DK (2000) Differential alteration in intestinal epithelial cell expression of toll-like receptor 3 (TLR3) and TLR4 in inflammatory bowel disease. Infect Immun 68:7010-7017.

Coats SR, Reife RA, Bainbridge BW, Pham TT, Darveau RP (2003) Porphyromonas gingivalis lipopolysaccharide antagonizes Escherichia coli lipopolysaccharide at toll-like receptor 4 in human endothelial cells. Infect Immun 71:6799-6807.

Duff GW, Durum SK (1983) The pyrogenic and mitogenic actions of interleukin-1 are related. Nature 304:449-451.

Elmquist JK, Scammell TE, Jacobson CD, Saper CB (1996) Distribution of Fos-like immunoreactivity in the rat brain following intravenous lipopolysaccharide administration. J Comp Neurol 371:85-103.

Faure E, Thomas L, Xu H, Medvedev A, Equils O, Arditi M (2001) Bacterial lipopolysaccharide and IFN- $\gamma$ induce Toll-like receptor 2 and Toll-like receptor 4 expression in human endothelial cells: role of NF- $\kappa \mathrm{B}$ activation. J Immunol 166:2018-2024.

Hohmann AG, Herkenham M (1999) Localization of central cannabinoid CB1 receptor messenger RNA in neuronal subpopulations of rat dorsal root ganglia: a double-label in situ hybridization study. Neuroscience 90:923-931.

Ivanov AI, Pero RS, Scheck AC, Romanovsky AA (2002) Prostaglandin $\mathrm{E}(2)$-synthesizing enzymes in fever: differential transcriptional regulation. Am J Physiol Regul Integr Comp Physiol 283:R1104-R1117.

Laflamme N, Rivest S (1999) Effects of systemic immunogenic insults and circulating proinflammatory cytokines on the transcription of the inhibitory factor $\kappa \mathrm{B} \alpha$ within specific cellular populations of the rat brain. J Neurochem 73:309-321.

Laflamme N, Rivest S (2001) Toll-like receptor 4: the missing link of the cerebral innate immune response triggered by circulating gram-negative bacterial cell wall components. FASEB J 15:155-163.

Laflamme N, Echchannaoui H, Landmann R, Rivest S (2003) Cooperation between toll-like receptor 2 and 4 in the brain of mice challenged with cell wall components derived from gram-negative and gram-positive bacteria. Eur J Immunol 33:1127-1138.

Lehnardt S, Lachance C, Patrizi S, Lefebvre S, Follett PL, Jensen FE, Rosenberg PA, Volpe JJ, Vartanian T (2002) The toll-like receptor TLR4 is necessary for lipopolysaccharide-induced oligodendrocyte injury in the CNS. J Neurosci 22:2478-2486.

Manthey CL, Perera PY, Henricson BE, Hamilton TA, Qureshi N, Vogel SN (1994) Endotoxin-induced early gene expression in C3H/HeJ (Lpsd) macrophages. J Immunol 153:2653-2663.

Oshikawa K, Sugiyama Y (2003) Gene expression of Toll-like receptors and associated molecules induced by inflammatory stimuli in the primary alveolar macrophage. Biochem Biophys Res Commun 305:649-655.
Poltorak A, He X, Smirnova I, Liu MY, Van Huffel C, Du X, Birdwell D, Alejos E, Silva M, Galanos C, Freudenberg M, Ricciardi-Castagnoli P, Layton B, Beutler B (1998) Defective LPS signaling in C3H/HeJ and C57BL/ 10ScCr mice: mutations in Tlr4 gene. Science 282:2085-2088.

Quan N, Whiteside M, Kim L, Herkenham M (1997) Induction of inhibitory factor $\kappa \mathrm{B} \alpha$ mRNA in the central nervous system after peripheral lipopolysaccharide administration: an in situ hybridization histochemistry study in the rat. Proc Natl Acad Sci USA 94:10985-10990.

Quan N, Stern EL, Whiteside MB, Herkenham M (1999) Induction of proinflammatory cytokine mRNAs in the brain after peripheral injection of subseptic doses of lipopolysaccharide in the rat. J Neuroimmunol 93:72-80.

Rehli M, Poltorak A, Schwarzfischer L, Krause SW, Andreesen R, Beutler B (2000) PU.1 and interferon consensus sequence-binding protein regulate the myeloid expression of the human Toll-like receptor 4 gene. J Biol Chem 275:9773-9781.

Reyes TM, Walker JR, DeCino C, Hogenesch JB, Sawchenko PE (2003) Categorically distinct acute stressors elicit dissimilar transcriptional profiles in the paraventricular nucleus of the hypothalamus. J Neurosci 23:5607-5616.

Rivest S (2003) Molecular insights on the cerebral innate immune system. Brain Behav Immun 17:13-19.

Song PI, Abraham TA, Park Y, Zivony AS, Harten B, Edelhauser HF, Ward SL, Armstrong CA, Ansel JC (2001) The expression of functional LPS receptor proteins CD14 and toll-like receptor 4 in human corneal cells. Invest Ophthalmol Vis Sci 42:2867-2877.

Turnbull AV, Rivier CL (1999) Regulation of the hypothalamic-pituitaryadrenal axis by cytokines: actions and mechanisms of action. Physiol Rev 79:1-71.

Vallieres L, Sawchenko PE (2003) Bone marrow-derived cells that populate the adult mouse brain preserve their hematopoietic identity. J Neurosci 23:5197-5207.

Wang JQ, McGinty JF (1995) Dose-dependent alteration in zif/268 and preprodynorphin mRNA expression induced by amphetamine or methamphetamine in rat forebrain. J Pharmacol Exp Ther 273:909-917.

Wehner T, Bontert M, Eyupoglu I, Prass K, Prinz M, Klett FF, Heinze M, Bechmann I, Nitsch R, Kirchhoff F, Kettenmann H, Dirnagl U, Priller J (2003) Bone marrow-derived cells expressing green fluorescent protein under the control of the glial fibrillary acidic protein promoter do not differentiate into astrocytes in vitro and in vivo. J Neurosci 23:5004-5011.

Whitfield Jr HJ, Brady LS, Smith MA, Mamalaki E, Fox RJ, Herkenham M (1990) Optimization of cRNA probe in situ hybridization methodology for localization of glucocorticoid receptor mRNA in rat brain: a detailed protocol. Cell Mol Neurobiol 10:145-157. 